\title{
Satellite guidance systems in agriculture: experimental comparison between EZ-Steer/RTK and AUTOPILOT/EGNOS
}

\author{
P. D'Antonio, C. D'Antonio, C. Evangelista, V. Doddato \\ School of Agricultural Sciences, Forest and Environmental, University of Basilicata, Potenza, Italy
}

\begin{abstract}
The research has been conducted using two different satellite-guidance devices and two different correction systems of the GPS signal: the EZ-Steer/RTK and Autopilot/EGNOS. The machines used in the tests were the tractor New Holland T7060, the rotary harrow Alpego DG-400 and the burier Forigo DG-45, in order to determine which of the two systems ensured the best quality of work. On the basis of the results obtained it is clear that the EZ-Steer/RTK system, guarantees a lower stability of the theoretical trajectory compared to the Autopilot/EGNOS system, above 1,77\%. From the elaboration of data of the two guidance systems behavior to manage the only width of transposition, it is observed that the EZ-Steer/RTK system is able to guarantee a better hold of the line compared to the Autopilot/EGNOS system, which provides a mistake of $164 \mathrm{~cm}$ on the total width of transposition and $2 \mathrm{~cm}$ on the mean value. In the matter of the normalized transposition surfaces, the Autopilot/EGNOS system ensures a better work quality.
\end{abstract}

\section{Introduction}

The precision farming is a form of agriculture multidisciplinary and technologically advanced, which recourses to machines equipped with "intelligent systems", able to dose the productive factors (fertilizers, pesticides, etc.) according to the real needs of the homogeneous areas constituent the plot (Verghagen and Bouma, 1997). The farming tends to manage every factor of production in varying measure, treating small areas inside the lot as separate surfaces. By doing so, the economic margin of crops can be increased, reducing the input of the technical means. Furthermore, the environmental impact and the quantity of the production factors used, such as pesticides and fertiliz-

Correspondence: C. D’Antonio, School of Agricultural Sciences, Forest and Environmental, University of Basilicata, via dell'Ateneo - 85100 Potenza, Itly. Tel.+39.0971205471, Fax: +39.0971205429,

E-mail: c_dantonio@yahoo.it

Key words: Precision farming, guidance assistance, transposition

CC Copyright P. D'Antonio et al., 2013

Licensee PAGEPress, Italy

Journal of Agricultural Engineering 2013; XLIV(s2):e34

doi:10.4081/jae.2013.s2.e34

This article is distributed under the terms of the Creative Commons Attribution Noncommercial License (by-nc 3.0) which permits any noncommercial use, distribution, and reproduction in any medium, provided the orig- ers, are significantly reduced. Therefore, the precision farming aims to adapt the contributions in a point manner, taking into account the local variability of the physical, chemical and biological characteristics of the field, and the timing of implant (Pierce and Sadler, 1997); at the same time, also the soil preparation work must be performed accurately, in order to avoid overlap, which would cause an increase of the costs. For this aim, a detailed mapping of the physical, chemical and biological properties of the field has been used, so that they can be managed by the control computer of the cultivation operations, placed beside the machines. Therefore, the implementation of the actions demands an automatic positioning system (GPS, Global Positioning System), which allows to the machine to recognize the exact location on the map, differentiating the agricultural operation to carry out. A group of 24 satellites in orbit around the earth forms the GPS. With three satellites, a receiver and through the three-dimensional triangulation, the receiver will indicate its position on the earth. That is done through the analysis of the high frequency signals, that the satellites send to the receiver, which calculates how long it takes to receive the signal and its position is displayed on a screen (Satellites 2006). In addition, it reduces the environmental damage and the risks in agriculture. During the crop production, the uncertainty of the yield may be reduced and the safety of the farmers incomes can be increased if the technological elements are used and combined correctly (Auernhammer 2001; Gandonou et al 2004; Chavas, 2008).

\section{Methods}

The experimentation has been carried out on two plots (A1 and A2), with temperatures around $22-25^{\circ} \mathrm{C}$ and a relative humidity (RH) between $60 \%$ and $65 \%$. The climate in this area is arid Mediterranean.

The surveys were conducted on two different satellite guidance devices using two different correction systems of the GPS signal:

- the EZ-Steer, with RTK precision system;

- the Autopilot, with EGNOS precision system.

The EZ-Steer is an assisted guidance system, wherein the management of the tractor direction happens by a motorized roll, placed beside the steering, which is operated by the control unit positioned in the tractor cabin. This system doesn't overcome the value of 90 points (on a scale that ranges from 50 to 150 ), because of a non-instantaneous reaction of the starter to the course correction pulses issued by the control unit and because of the roll slipping light on the wheel; it was observed that, using a precision RTK and setting a value more than 90 points, the EZ-Steer system get in overcorrection, since the course corrections operated by the control unit, called in this article T2, can't be handled by the roll placed on the wheel and for this the tractor is not perfectly able to follow the direction assigned through GPS, but it accentuates the sine frequency around this. The Autopilot, instead, is an integrated system, which uses the hydrostatic guidance system for the management of the tractor through a hydraulic control unit, which 
communicates with the control unit, called in this article, T3, placed in the control room. The control unit T3 communicates, both, with the GPS antenna and with the sensors proximal to the wheels, which indicate how the feed axis divert respect to the orthogonal of the tractor. The Autopilot's hydraulic circuit, differently from the EZ-Steer, allows to work with a value up to 135 points before going in overcorrection, that means it has a remarkable ability to maintain the correct trajectory also on land full of holes and/or soft. The GPS with EGNOS error correction can obtain a positioning accuracy, that is a precision between consecutive passes, of $20 \mathrm{~cm}$ and a repeatability year after year of $90 \mathrm{~cm}$ (it's a free service); the RTK, instead, is a high-precision technology, with an accuracy between consecutive passes of $2.5 \mathrm{~cm}$ and a repeatability year after year of $2.5 \mathrm{~cm}$. The RTK is able to provide an elevation accuracy of $5 \mathrm{~cm}$, with an improvement of almost two orders of height compared to the GPS standard (DGPS), since this system is able to extract the additional information, examining the carrier wave of the GPS signal (Tyler et al., 1997). The RTK system's disadvantages are: a higher cost, the need to establish a local base station and a significant decrease compared to the GPS standard methods. A light-bar provides information of the visual guide to the operator, who may make some corrections of manual steering (Trimble Navigation, Ltd., 2005). An assisted steering system makes automatic these adjustments and the operator directs only the tractor and his intention to turn at the end of the field (Grisso and Alley, 2002).

For the tests, the tractor used was the New Holland T7060 and as machines the rotary harrow, Alpego DG-400 and the buriers Forigo DG45.The operating machines have been used to improve the quality of work, understood as a shorter overlap or a shorter deviation between wipes contiguous. The tractor New Holland T7060 is operated by a NEF motor, which has been designed to minimize the vibrations. It is a 6cylinder engine capable of developing a stable power at any speed. In addition, this engine has a low speed that reduces the noise and the vibrations. The harrow Alpego DG-400 is a folding rotary harrow, used to break up the clods after the plowing to prepare the seeding or planting bed. It has a width of $4.10 \mathrm{~m}$ and a work front of $4.00 \mathrm{~m}$. It is a harrow that can fold on itself turning into a little shape, below $2.50 \mathrm{~m}$ in width. With regard to the harrowing depth, the value set up was $10 \mathrm{~cm}$; a sampling was performed and the related data were shown in the table 6 . The buriers is a machine used for the preparation of the seedbed on soil with a good supply of skeleton, allowing to obtain a surface layer well refined. Its mechanical action is due to the rotation impressed by the power takeoff tractor to a rotor with vertical teeth (knives) type milling cutter. In front, the Forigo DG45 - 400 is provided with a pair of the conveyor discs, which represent the most protruding part of the operating machine, characterizing the amplitude of the work front. The researches carried out on flat surfaces, so that the rover and the eventual antenna RTK could be used by the largest number of satellites (in fact, leaning both to GLONAS and to GPS, it has been detected on the user interface display, placed in the driver's cab, a medium of 10 satellites); for the same reason we worked on flat areas without the presence of trees, power lines, farms or similar facilities, which would block out the signal in case it interposes between the receiver and the satellite. The surveys have been performed using a tape measure of $20 \mathrm{~m}$, a tape measure of $3 \mathrm{~m}$, steel rods, a carpentry line and a land surveyor squaring. Between two passes perfectly contiguous, it can see the entire line of juncture, which in the absence of transpositions, keeps perfectly linear along the entire route. Combining the line of juncture with the land surveyor squaring thread and working backwards through armor rods, it was possible to align and stake out the extremes of the linear transpositions along the joint. Then, it was detected the distance between the stakes, obtaining the so-called length of transposition and also connecting the two pegs with a line, it was possible to detect the maximum distance, identifying in this way, the width of transposition.
The surveys were performed over several days in the countryside on a route of $13.000 \mathrm{~m}$ and 80 observations for each plot. The basic parameters that have characterized the two test scenarios are summarized in the Table 1 and in the Table 2 there are the machine operating data.

The surveys have been performed on pairs of contiguous swaths, to identify areas where happen an overlap or some variations, so that even the measure results reasonable.

The principle is as follows: let us suppose that the work front amplitude of the operating machine is fixed, for example of $4.00 \mathrm{~m}$ for the Alpego DG-400, it follows that two swaths pull in parallel between them, will show a constant amplitude of $8.00 \mathrm{~m}$, for which any section (orthogonal to the advance direction of the tractor) will not show neither overlaps or derivations, and therefore, the satellite guidance system will have done a good work. In the overlap areas we will have a reduction of this width, which will be of value below $8.00 \mathrm{~m}$, for example, it will be of value $7.50 \mathrm{~m}$. Therefore, the lateral transposition datum reported on the land book will be the result between the width measured in the field, in this case $7.50 \mathrm{~m}$, and the nominal width of the two contiguous swaths, in the case $8.00 \mathrm{~m}$. The transposition length, that is the transposition entity in the direction of tractor advancement, was measured detecting the distance between the point in which the tractor deflected the ideal trajectory and the point where the tractor inserted again on the ideal trajectory. In the areas of deviation, instead, the front is greater than the front that would occur if the swaths were maintained tangential, for which, in this case the lateral transposition datum reported on the land book will be the result of the difference between the width actually measured in the field, for example $8.50 \mathrm{~m}$, and the nominal width of the two adjacent swaths, $8.00 \mathrm{~m}$. For each transposition, then, has been calculated the apparent area, multiplying the transposition length for its width. Obviously, since in the variance analysis for one factor (ANOVA) it is necessary to standardize the number of the detected data, to the non-existent data have been assigned a value of 0 and the average was calculated considering these terms.

Table 1. Basic nominal geometric parameters. E/L-Steer/RII Autopilot/EGNOS

\begin{tabular}{lcc} 
A swath average length(m) & 250 & 271 \\
Total number of passes made & 52 & 48 \\
\hline Meters travelled (m) & 13000 & 13000 \\
Contiguos passes number & 26 & 24 \\
\hline Single pass width (m) & 3,8 & 4 \\
Two contiguous passes width (m) & 7,6 & 8 \\
\hline Swath total width (m) & 197,6 & 192 \\
Two contiguos passes surface (m2) & 1900 & 2168 \\
\hline Dof work surface (m2) & 49400 & 52032 \\
\hline
\end{tabular}

Table 2. Machine operative data.

\begin{tabular}{lcc} 
& $\begin{array}{c}\text { Forigo DG45 + } \\
\text { New H. T7060 }\end{array}$ & $\begin{array}{c}\text { Alpeg0+ } \\
\text { New H. T7060 }\end{array}$ \\
Average speed (Km/h) m/min) & 3,$6 ; 60$ & 4,$0 ; 66,7$ \\
Work front width (m) & 3,8 & 4 \\
\hline Processing depth (m) & 0,2 & 0,1 \\
Fuel consumption (l/h) & 15,4 & 15,8 \\
\hline
\end{tabular}




\section{Results and discussion}

From the elaboration of data in relation to the behavior of the two satellite guidance systems, that manage the only direction of tractor advancement, we have obtained the following results (Table 3).

On the basis of these results you gather that the EZ-Steer/RTK system ensures a lower capacity of the theoretical path, in fact, the total of the travelled route shows an error of $229.42 \mathrm{~m}$ respect to the Autopilot/EGNOS system, above $1.77 \%$. From the elaboration of data relating to the behavior of the two guidance systems in manage only the transposition width, we have obtained the following results (Table 4). So it can be observed that the EZ-Steer/RTK system ensures a better approximation of the tractor trajectory to the theoretical path respect to the Autopilot/EGNOS system, which provides an error greater than 1.64 $\mathrm{m}$ over the total transposition width and $2 \mathrm{~cm}$ over the average transposition width. This is because the RTK, proposing a greater precision lead the EZ-Steer system to approach more to the theoretical path, differently from the EGNOS system, which manages an accuracy of $20 \mathrm{~cm}$, therefore, despite the Autopilot control ability on the tractor, at the end you can get more lateral transposition. As regards the latitudinal parameter, unlike the transposition length, has not been considered the percentage of the transposition width respect to the total of the work front, in that, at least in this location, not actually being in possession of an adequate amount of data, the transposition width and the total width of the work front are not connected with each other; it follows that, the total work front is not derived from the quality of latitudinal work and the use of two machines has been exclusively as tracer, because all this, the piloting, being automated, will repeat the lateral

Table 3. Transposition length results.

\begin{tabular}{|c|c|c|c|}
\hline & Steer/RTK & Autopilot/EGNOS & VARIAZIONE \\
\hline Swath average length (m) & 250 & 271 & -21 \\
\hline Route travelled (m) & 13000 & 13000 & 0 \\
\hline Transposition totallength (m) & 998,64 & 769,22 & 229,42 \\
\hline Transposition average length ( & 12,483 & 9,6153 & 2,8677 \\
\hline $\begin{array}{l}\text { Transposition length of total } \\
\text { travelled route (\%) }\end{array}$ & 7,682 & 5,917 & 1,77 \\
\hline Length min. of transp(m) & 1,3 & 2,45 & $-1,15$ \\
\hline Length max oftransp(m) & 28,7 & 17,1 & 11,6 \\
\hline
\end{tabular}

Tabella 4. Results about transposition width.

\begin{tabular}{|c|c|c|c|}
\hline EZ & Steer/RTK & Autopilot/EGNOS & VARIAZIONE \\
\hline Work front widh (m) & 3,8 & 40,2 & \\
\hline Two passes contiguous width (m) & 7,6 & 80,4 & \\
\hline Swath width(m) & 197,6 & $192-5,6$ & \\
\hline Total transposition width(m) & 11,6 & 13,237 & 1,637 \\
\hline Average transposition width (m) & 0,145 & 0,165 & 0,02 \\
\hline Length min. of transp(m) & 0,05 & 0,08 & 0,03 \\
\hline Length max.oftransp(m) & 0,355 & 0,375 & 0,02 \\
\hline
\end{tabular}

transposition error despite you will work on $3.80 \mathrm{~m}, 4.00 \mathrm{~m}$ to $20.00 \mathrm{~m}$. From the elaboration of data relating to the behavior of the two guidance systems in managing the areas of normalized transposition, we obtained these results (Table 5).

About these values is possible to observe that the Autopilot/EGNOS system is capable of ensure a better quality of work than the EZSteer/RTK system, in fact, it provides a precision on the transposition total area, equal to $6.396 \mathrm{~m}^{2}$. The area of transposition, therefore, is the parameter that most of all allows to compare the two combinations of guidance systems, as it is a datum that merges the longitudinal behavior of transposition with the latitudinal one. As for the transposition width also for the surface of transposition is not considered the proportion (\%) of the transposed area respect to the total of worked area, in that, with increasing of the work front, and therefore, the surface unitarily worked, the surface transposed being equal, it follows its reduction if we consider it in percentage terms.

As you can see the average depth of work respects the operating nominal depth when the tractor is supported either by the system EZSteer/RTK or by the Autopilot/EGNOS system. The variance analysis (ANOVA) affected parameters such as: transposition length, transposition width and normalized areas. For the calculation of the variance of the transposition length we have analyzed the data obtained, which have reported in the graph relative to the transposition lengths provided by the two guidance systems (Figure 1).

The results obtained showed that the values of transposition length surveyed in the field for the EZ-Steer/RTK and the Autopilot/EGNOS systems one deviate significantly from the average. Similarly to the calculation of the variance of the transposition width have been analyzed the data collected which have shown in the graph of the transposition

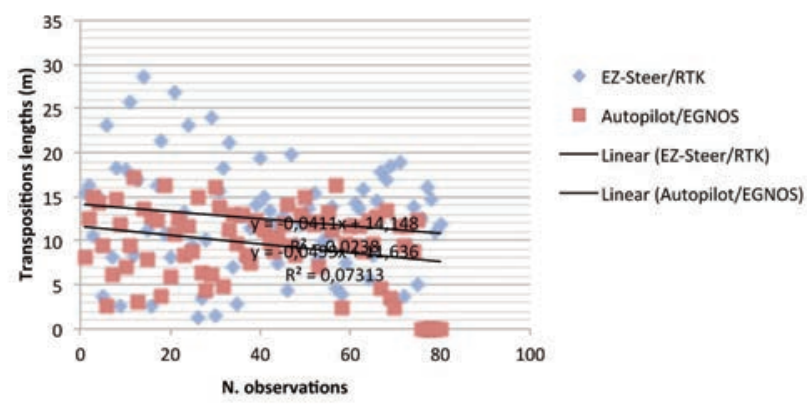

Figure 1. Transposition lengths provided by the two guidance systems.

Table 5. Results on the surface of transposition.

BL-Steer/RTK Autopilot/EGNOS VARTAZIONE

\begin{tabular}{lccc} 
Swath average length (m) & 250 & 271 & -21 \\
Route travelled (m) & 13000 & 13000 & 0 \\
\hline Transposition totallength (m) & 998,64 & 769,22 & 229,42 \\
Transposition average length (m) & 12,483 & 9,6153 & 2,8677 \\
\hline $\begin{array}{l}\text { Transposition length } \\
\text { of total travelled route (\%) }\end{array}$ & 7,682 & 5,917 & 1,77 \\
Length min. of transp(m) & 1,3 & 2,45 & $-1,15$ \\
\hline Length max oftransp(m) & 28,7 & 17,1 & 11,6 \\
\hline
\end{tabular}


widths provided by the two guidance systems (Figure 2.).

For the calculation of the variance of the transposition normalized areas, obtained by multiplying for each survey, the transposition length for its width, we have analyzed the collected data which have shown in the graph (Figure 3 ).

The variance analysis on the normalized areas shows that the values obtained from the systems EZ-Steer/RTK and Autopilot/EGNOS do not deviate significantly from the average.

The survey results are summarized in the following table (Table 7) to deduce the behavior of the two systems and draw conclusions.

In longitudinal terms, a good work quality is given by the null transposition length. It's clear from the comparison with the data that the EZ-Steer/RTK system, provides a lower capacity of the theoretical trajectory. In fact, the total of the carried out route, shows a longitudinal transposition of $229.42 \mathrm{~m}$ more than the Autopilot/EGNOS system, equal to $1,77 \%$ of the $13,000 \mathrm{~m}$ routes. It follows that, between the two systems, the Autopilot/EGNOS is able to correct before and better the deviation from the theoretical route. The device EZ-Steer is an assisted guidance system and the tractor is operated by a motorized roller, whose management is assigned to the control unit T2 placed in the tractor's cab. The starter, which is the limit of the EZ-Steer system, is unable to handle the multiple impulses of course correction issued by the control unit causing a light slipping on the wheel, reducing the system sensitivity. All these elements are summarized from the parameter aggressiveness, which expresses the ability of the system to allow the tractor to point out the theoretical trajectory. Increasing the aggressiveness the systems helps the tractor to follow a rectilinear profile and faithful to the theoretical trajectory. It exists, however, a limit called overcorrection that is proportional to the architecture of the system. The EZ-Steer, in fact, does not allow to go over 90 points of aggressiveness, because exceeding this threshold it is in overcorrection and

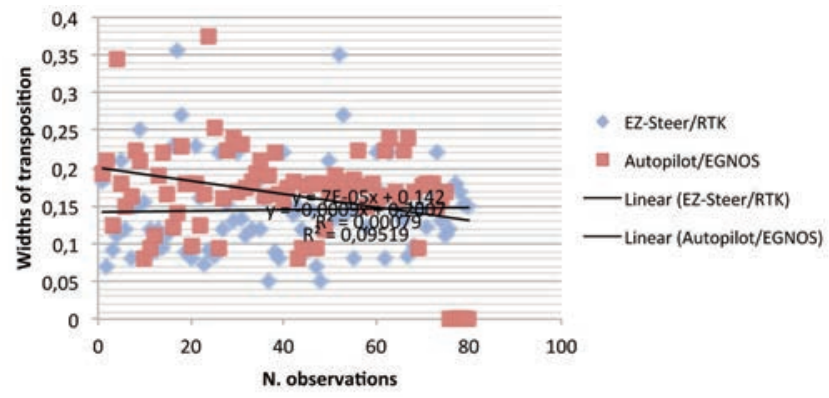

Figure 2. Comparison between the transposition widths provided by the two guidance systems.

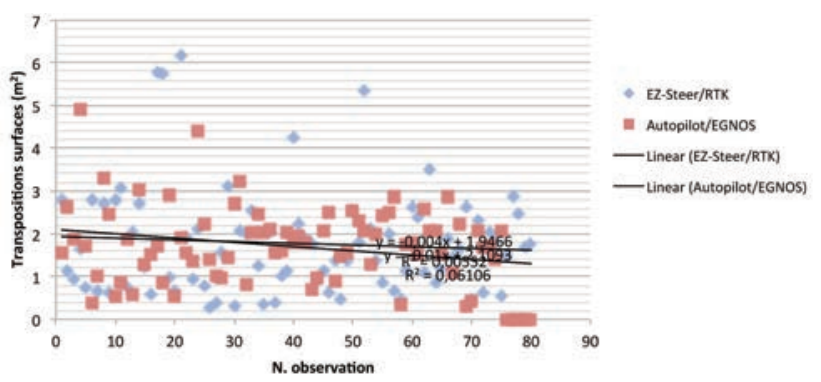

Figure 3. Comparison between the transposition normalized surfaces provided by two guidance systems.

instead to follow the theoretical trajectory increases the error around it. This implies a greater transposition length, and, therefore, a lower quality of work. The Autopilot system, instead, allows to reach an aggressiveness of 135 points, justifying the different length of transposition, found in the experimental stage, between the two systems. The EZ-Steer system, however, differently from the Autopilot, is implemented by a correction RTK that according the test it should have offset the reduced aggressiveness, and therefore, it would have to reduce the transposition length but it was not like. In fact, the EZ-Steer/RTK system has found a transposition length equal to $998.64 \mathrm{~m}$, while the Autopilot/EGNOS system has found a transposition length of $769.22 \mathrm{~m}$. The control ability of the actual trajectory on the ideal trajectory and the aggressiveness of the two guidance systems, the EZ-Steer and the Autopilot prevail against the accuracy correction of the satellite signal took place by the RTK and EGNOS devices. The results on the transposition widths point out the different ability of the precision correction accomplished by both systems. While, with the Autopilot/EGNOS system has a transposition average width equal to $16.5 \mathrm{~cm}$, the EZ-Steer/RTK system has a transposition average width equal to $14.5 \mathrm{~cm}$. For the combination EZ-Steer/RTK, the $14.5 \mathrm{~cm}$ are, however, a result that does not respect the power of the RTK correction $(2.5 \mathrm{~cm})$, while the Autopilot/EGNOS combination is fully integrated within the $20 \mathrm{~cm}$ guaranteed by EGNOS correction. The system Ez-Steer/RTK's transposition average width $(14.5 \mathrm{~cm})$ does not respect the potential of the RTK correction $(2.5 \mathrm{~cm})$, while, the system Autopilot's transposition average width is included in the correction EGNOS's $20 \mathrm{~cm}$. The motivation, also in this case, is referable to the different aggressiveness of the two satellite guidance systems, this is because a 90 points' aggressiveness induces the tractor to move away from the ideal trajectory, unlike what happens with a 135 points' aggressiveness obtained by the Autopilot system. Comparing the two systems on the basis of the transposition

Table 6. Results on the work depth of the machines.

\begin{tabular}{lcc} 
& Forigo DG45 & Alpego DG - 400 \\
Work nominal depth (m) & 0,2 & 0,1 \\
Work total depth (m) & 16,078 & 8,002 \\
\hline Work average depth (m) & 0,200975 & 0,100025 \\
\hline
\end{tabular}

Table 7. Results about the performances of two guidance systems. E/-Steer/RTK Autopilot/EGNOS VARIAZIONE

\begin{tabular}{lccc} 
Transposition total length (m) & 998,64 & 769,22 & 229,42 \\
Transposition average length (m) & 12,483 & 9,6153 & 2,87 \\
\hline $\begin{array}{l}\text { Variance-Transposition average length } \\
\text { Percentage of the transposition length }\end{array}$ & 38,3352 & 18,3766 & 19,96 \\
on the total of travelled route (\%) & 7,682 & & \\
\hline Transposition total width (m) & 11,6 & 5,917 & 1,77 \\
Transposition average width (m) & 0,145 & 13,237 & $-1,64$ \\
\hline Variance - Transposition average width & 0,0037 & 0,165 & $-0,02$ \\
Transposition total surface(m $\left.{ }^{2}\right)$ & 142,672 & 0,0043 & 0,00 \\
\hline Transposition average surface(m $\left.{ }^{2}\right)$ & 1,7834 & 136,276 & 6,40 \\
Variancetransposition average surface & 1,5861 & 1,7035 & 0,08 \\
\hline
\end{tabular}


normal areas show that both are characterized by a good reliability that is even higher in the case of the Autopilot/EGNOS association, able to contain the transposition surface of only $6.396 \mathrm{~m}^{2}$, thanks mainly to the lower longitudinal transposition. As you can see, the machines using the two systems, the Autopilot/EGNOS and the EZ-Steer/RTK had an homogeneous regularity of work. These results, however, can be attributed to the capability of the tractor New Holland T7060's active suspensions and to the regularity of the surfaces which have not produced a considerable pitch stress to the tractor. The regular work depth doesn't result from the control units of control, because although the T3, compared to T2, also manages the pitch, this capacity has to be understood in terms of the tractor's apparent position respect to the real position and therefore it has to be understood in terms of geo-referencing, not mechanical. There will be different results from the ones found when the control unit T3 have planned with a pitching control that operates on the suspension hydraulic circuit.

\section{Conclusions}

The conducted experimentation shown the way the two satellite guidance systems examined don't have a significant differences in the quality of work, although the Autopilot/EGNOS system has a greater reliability on the transposition length. It follows that the two combinations of satellite guidance, in view of the similar amount of latitudinal transposition, are suitable for all those jobs which tolerate some mistakes, such as: plant protection treatments, fertilizing, harrowing, mowing, reaping and so on, while for a high precision work, such as the sowing, weeding, planting, processing among rows, there is the need to use a combination that guarantees a maximum precision, and for this it will be necessary to optimize the system Autopilot supporting by the RTK correction. The maximum precision that comes from, however, can entail the saving on factors of production and this solution would be hoped for the increase of their purchase cost and for the growth of company. An entrepreneur who wants the growth of his company, adopting the two systems must consider several aspects, such as: the current size and the expansion potential of company, the crops ordering current and future, the possibility of entering in the subcontracting's business, the EZ-Steer's versatility compared to the Autopilot, but also the Autopilot's precision potentials against the EZ-Steer and therefore an actual concrete opening towards a thrust precision farming. The disadvantage of the Autopilot and that since it has the integrated system, it can be disassembled by a tractor and the reassemble on another one which may be suitable for the performed work. These systems make independent the tractor feed from the operator's view; this is an advantage for the operator who will have a less workload and more time to focus his attention on what it is important, that is, the processing. These are systems that allow to reduce the overlaps. This condition implies a better quality of work, also motivated by a reduced compaction of land (for minor steps), by an increase in fertility, by a reduction of erosion, by a less disturbance to crops in progress, with a higher productivity of the yards, thanks to the use of the front work, and the decrease of passes number being equal surface and a greater speed of execution. This in turn, entails a decrease in costs, a greater productivity and a better management of company. The satellite guidance sys- tems, in fact, allowing the tractor in the field to geo-reference, which are validly supported by remote sensors, from information derived from remote sensors and therefore by the prescription maps (which quantify the intensity of interventions in various areas of the field), in conjunction with appropriate technologies, which allow you to practice the VRA (variable distribution of inputs with sensors), find step by step the physical-chemical state of the soil, the nutritional state of the crop, the plant health state of the same, and the degree of production maturation etc.. An further advantage of these systems is a less pollution of water and air and a traceability of products, in fact, the geo-referencing, supported by the context of precision agriculture, identifies the exact point of origin of the product and the treatments to which it was subjected. The EZ-Steer's advantage regards the possibility of being applied to over several tractors, in fact, within a couple of hours it can be disassembled by a machine and reassemble on another one. The Autopilot, instead, is an integrated system, placed on the guidance hydraulic circuit, which greatly improves the precision of the assisted driving (in fact, you get to 130 of aggressiveness), at the same time has the disadvantage of not be able to be moved by a tractor to another one, and therefore, to a greater precision of the guidance system there is the cost of a less versatility. The disadvantages are: a precision work not very good, the occasional loss of the satellite signal (attributable to several factors: power lines, trees, houses, water openings for irrigation, etc..), and a substantial purchase for the systems more advanced.

\section{References}

Auernhammerr H. Precision farming - the environmental challenge. Computer and Electronics in agriculture 2001; 31:43- 30.

Chavas J. P. A cost approach to economic analysis under state-contingent production uncertainty. American Journal of Agricultural Economics. Blackwell Publishing Co 2008; 435: 446 - 90 (2).

Gandonou, J M, Dillon C, Harman W, Williams J. Precision farming as a tool in reducing environmental damages in developing countries: a case study of cotton production in Benin. American Agricultural Economics Association 2004. Annual Meeting. Available from:http://ageconsearch.umn.edu/bitstream/20086/1/sp04ga02.pd

Grisso R. and M. Alley. (2002). Precision farming tools - light bar navigation. Publication 442-501. Blacksburg: Virginia Cooperative Extension Service

Pierce F.J., Sadler E.J. (1997). The state of site specific management for agriculture, ASA Publ., ASA, CSSA e SSSA, Madison, WI, USA.

Tyler D A,. Roberts D W, Nielsen G A. (1997). Location and guidance for site-specific management. In: The State of Site-Specific Management for Agriculture1997. ASA, CSSA, SSSA, Madison, WI; 1997. pp. 161-181.

Trimble Navigation, Ltd. EZ SteerTM System reference guide. Overland Park, Kan; 2005.

Verhagen J , Bouma J, 1997. "Modeling soil variability”. In Pierce F.J., Sadler E.J. (ed).The state of site specific management for agriculture 1997: ASA Publ., ASA, CSSA e SSSA, Madison, WI, USA; 1997. 